\title{
Infliximab-Induced Interstitial Lung Disease in a Patient With Psoriatic Arthritis
}

\author{
Lan Quang, MD, Anthony Scarpaci, MD
}

\section{Introduction}

Infliximab (Remicade, Centocor, Inc., Malvern, PA), a chimeric monoclonal antibody derived from both murine and human antibody sequences and directed against TNF- $\alpha$, is one of the disease modifying anti-rheumatic drugs (DMARDs) used in the treatment of psoriatic arthritis and other autoimmune inflammatory conditions such as rheumatoid arthritis, ankylosing spondylitis, and Crohn's disease. It is commonly used in combination with methotrexate for increased efficacy and reduction of the development of anti-infliximab antibodies. A concerning feature of infliximab therapy is its association with an increased risk of infection or reactivation of diseases that can cause pulmonary complications, most notably tuberculosis and fungal infections. Additionally, there are a number of patients who have developed non-infectious interstitial lung disease following treatment with infliximab. These events usually occur after the second to third infusions and can be preceded by a course of methotrexate. In the setting of prior methotrexate therapy, it is suggested that infliximab may potentiate the manifestations of pulmonary toxicity due to methotrexate, specifically methotrexate pneumonia. ${ }^{1}$ In the following case report, we cite an example of the development of interstitial lung disease after infliximab therapy in a patient with psoriatic arthritis.

\section{Case Presentation}

A 59-year-old female with a previous medical history of asthma and psoriatic arthritis with recent infliximab therapy presented to the Emergency Department (ED) with a chief complaint of progressive shortness of breath for two weeks. She experienced chest tightness and dyspnea with exertion. She denied wheezing and received minimal relief with nebulizers. She also stated the presence of subjective fevers and sweating that started two weeks ago. She denied leg swelling, weight gain, recent trips, or sick contacts. Upon examination, she had difficulty speaking due to shortness of breath and had rales on her left lung. No wheezing or jugular venous distention was noted. She required 2 liters of oxygen by nasal cannula to maintain her saturations at $96 \%$. Cardiac examination was within normal limits. The rest of the patient's exam was normal. Initial chest $\mathrm{x}$-ray was read as prominence of interstitial lung markings.

Additional pertinent medical history includes: psoriatic arthritis diagnosed 7 months prior to admission for which she had received prior treatment. She had received intra-articular steroid injections into the hand joints. After that therapy did not relieve her symptoms she received two doses of weekly methotrexate. This therapy was discontinued due to patient intolerance. The patient began infliximab therapy three months later. She had received three infusions of infliximab on a biweekly schedule. The last infusion was administered two weeks prior to admission. Her medications at presentation included: metoprolol succinate (XL) $50 \mathrm{mg}$ po qday, fenofibrate $145 \mathrm{mg}$ po qday, omega-3-fatty acid $4 \mathrm{~g}$ po qday, irbesartan $150 \mathrm{mg}$ po qday, ezetemibe $10 \mathrm{mg}$ po qday, rosuvastatin $20 \mathrm{mg}$ po qday, diphenhydramine $50 \mathrm{mg}$ po qhs, furosemide $20 \mathrm{mg}$ po qMWF, fluticasone/salmeterol 250/50 mcg diskus day, alprazolam $1 \mathrm{mg}$ po qhs PRN, oxycodone/acetaminophen $5 / 325 \mathrm{mg}$ po $\mathrm{PRN}$, and albuterol nebulizers PRN. The patient stated that she develops seizures from gabapentin.

The patient's medical and surgical histories also included: HTN, hyperlipidemia, mitral valve prolapse, history of supraventricular tachycardia, anxiety, steroid-induced diabetes mellitus, steroid-induced psychosis, thyroid cysts, MRSA pneumonia, ocular surgery, ulnar nerve surgery, and breast lump removal. Her social history is significant for drinking 2-3 alcoholic beverages per week and no use of tobacco or recreational drugs. She is normally fully functional and lives alone. She works for a newspaper publisher.

Pertinent positives on review of systems included: chest tightness, shortness of breath, cough, hoarseness, and epistaxis. Pertinent negatives on review of systems included: no sputum production, wheezing, edema.

\section{Assessment}

In the $\mathrm{ED}$, the patient was treated with albuterol nebulizers. After no improvement in her symptoms, the patient was admitted to telemetry for further cardiac and respiratory evaluation. Acute MI was ruled out with three negative troponins and an unremarkable EKG. Echocardiogram showed normal left ventricular size and function, an ejection fraction of 70 percent, and mild impaired relaxation of the left ventricle. Her symptoms did not resolve with supplemental oxygen and nebulizer treatment. Moxifloxacin was started for empiric antibiotic coverage for community-acquired pneumonia. Room air arterial blood gas was within normal limits. Despite this laboratory value, the patient desaturated while ambulating on room air to $77 \%$. Chest CT (Figure 1) showed interstitial lung disease with mild to moderate congestive heart failure. Despite diuresis with furosemide, the patient's symptoms did not resolve so bronchoscopy was performed. Bronchoalveoloar lavage was negative for malignant cells, PCP, fungus, and mycobacterium. Transbronchial biopsy (Figure 2) was consistent with active pneumonitis with interstitial inflammation, edema, and fibrosis. Air spaces were lined by type II pneumocytes and contained macrophages, fibrin and organizing pneumonia, which are features consistent with drug-induced pneumonitis.

\section{Management}

The patient's radiologic studies and pathology are consistent with drug-induced pulmonary toxicity. After extensive review of her medications, it was felt that the only drug that was temporally related to the patient's symptoms was 
infliximab. After nebulizers, antibiotics, and diuresis failed to provide symptom relief, the patient was started on prednisone $50 \mathrm{mg}$ orally daily. She was discharged 7 days after admission on a prednisone taper and maintained on two liters portable oxygen with mild improvement in her symptoms.

\section{Discussion}

Recent literature has implicated infliximab in the development of interstitial pneumonitis. ${ }^{1}$ Many of the cases are in patients with rheumatoid arthritis or Crohn's Disease. ${ }^{1,2}$ Often, rheumatoid arthritis patients are concurrently receiving methotrexate therapy to maximize disease modification. Notably, the pulmonary toxicity can be quite severe. A prior case series reports four patients with interstitial pneumonia and one patient with bronchiolitis obliterans organizing pneumonia following infliximab therapy. The patients had rheumatoid arthritis and were not treated with methotrexate due to preexisting lung disease. Each of the patients except the patient with bronchiolitis obliterans experienced rapidly declining hospital courses and eventually expired from respiratory failure despite steroids and ventilatory support. ${ }^{3}$

The pathophysiology of infliximab induced pneumonitis is not known. One possible theory is that infliximab may potentiate the pulmonary toxicity of methotrexate. However, this does not account for patients who were not receiving methotrexate therapy. Animal models of bleomycin-induced lung toxicity have shown that blocking TNF- $\alpha$ worsens toxicity. Other research has shown that inhibition of TNF- $\alpha$ may increase proinflammatory cytokines. Additionally, when infliximab binds to TNF$\alpha$ on CD-4+ T cells and macrophages, proteolytic enzymes are released which can lead to pulmonary damage. ${ }^{3}$

This is the first reported case of interstitial pneumonitis due to infliximab in the psoriatic arthritis population. Our patient's exposure to methotrexate was limited to only a short treatment of two doses, which was terminated secondary to intolerance. Thus, the lack of a prior therapeutic methotrexate regimen and the temporal relationship of the development of lung symptoms with the start of infliximab infusion suggest that infliximab alone may have induced interstitial lung disease.

As pulmonary complications associated with infliximab are further documented, it becomes important to screen patients for risk factors for pneumonitis prior to initiating therapy. Some of the identified risk factors specifically for methotrexate pneumonitis include preexisting lung disease, older age, diabetes, hypoalbuminemia, and previous use of DMARDs. ${ }^{4}$



Figure 1. High Resolution Chest CT: Cross-section demonstrating diffuse bilateral groundglass opacities and pulmonary edema.

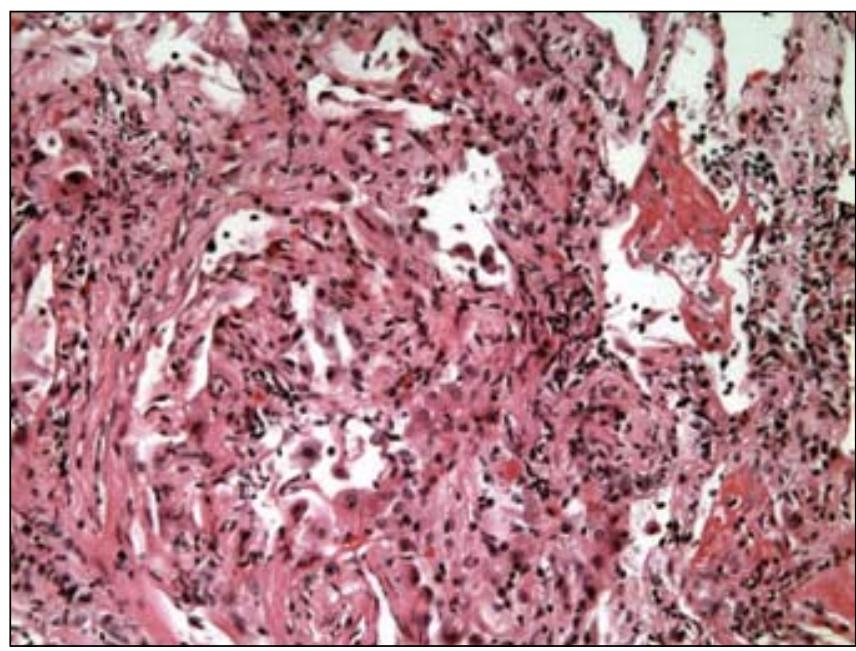

Figure 2. H\&E stain of tissue sample from transbronchial lung biopsy. The above slide demonstrates active pneumonitis with interstitial inflammation, edema, and fibrosis. Airspaces are lined by type II pneumocytes and contain macrophages, fibrin and organizing pneumonia.

\section{References}

1. Villeneuve E, St-Pierre A, Haraoui B. Interstitial Pneumonitis Associated with Infliximab Therapy. Journal of Rheumatology 2006; 33: 1189-1193.

2. Weatherhead, M., et al. Interstitial Pneumonitis after Infliximab therapy for Crohn's Disease. Inflamm Bowel Dis 2006; 12: 427-428.

3. Ostor AJ, Chilvers ER, Somerville MF, Lim AY, Lane SE, Crisp AJ, Scott DG. Pulmonary Complications of infliximab therapy in patients with rheumatoid arthritis. Journal of Rheumatology 2006; 33: 622-628.

4. Mori S, Imamura F, Kiyofuji C, Sugimoto M. Development of interstitial pneumonia in a rheumatoid arthritis patient treated with infliximab, an antitumor necrosis factor $\alpha$-neutralizing antibody. Mod Rheumatol 2006; 16: 251-255. 